\title{
A CHARACTERIZATION OF TORSIONFREE MODULES OVER RINGS OF QUOTIENTS
}

\author{
JOHN A. BEACHY
}

\begin{abstract}
Let $\sigma$ be an idempotent kernel functor defining the ring of left quotients $Q_{\sigma}(R)$. We introduce a notion of $\sigma$-divisibility, and show that a $\sigma$-torsionfree $R$-module $M$ is a module over $Q_{\sigma}(R)$ if and only if $M$ is $\sigma$-divisible.
\end{abstract}

In his paper Rings and modules of quotients, Goldman [2] investigated the construction of rings of quotients of a ring $R$ with respect to idempotent kernel functors of the category ${ }_{R} M$ of left $R$-modules. If $\sigma$ is such an idempotent kernel functor, then it was shown that every $\sigma$-torsionfree and $\sigma$-injective left $R$-module is a left module over the ring of quotients $Q_{\sigma}(R)$. We have called a module ${ }_{R} M \sigma$-divisible if it is generated in the categorical sense by the $R$-module $Q_{\sigma}(R)$. In Theorem 4 below we show that a $\sigma$ torsionfree module ${ }_{R} M$ is a left $Q_{\sigma}(R)$-module (extending the action of $R$ ) if and only if $M$ is $\sigma$-divisible. In Theorem 6 we show that the quotient functor $Q_{\sigma}$ is naturally isomorphic to the functor $Q_{\sigma}(R) \otimes \_$if and only if every $\sigma$-torsionfree and $\sigma$-divisible left $R$-module is $\sigma$-injective.

All rings considered in this note are associative rings with identity, and all modules are assumed to be unital. For the convenience of the reader we repeat the relevant definitions of [2] and [3]. A functor $\sigma$ from ${ }_{R} \boldsymbol{M}$ into itself is called a kernel functor if it has the following properties: (i) $\sigma(M)$ is a submodule of $M$ for every $M \in{ }_{R} M$. (ii) If $f: M \rightarrow N$ is a homomorphism, then $f(\sigma(M)) \subseteq \sigma(N)$, and $\sigma(f)$ is the restriction of $f$ to $\sigma(M)$. (iii) If $M^{\prime}$ is a submodule of $M$, then $\sigma\left(M^{\prime}\right)=M^{\prime} \cap \sigma(M)$. If, in addition, $\sigma(M / \sigma(M))=0$ for all $M \in{ }_{R} M$, then $\sigma$ is called an idempotent kernel functor.

For the remainder of the note we let $\sigma$ be a fixed idempotent kernel functor of ${ }_{R} \boldsymbol{M}$. A module ${ }_{R} M$ is called $\sigma$-torsion if $\sigma(M)=M$; it is called $\sigma$-torsionfree if $\sigma(M)=0$. A submodule $M^{\prime}$ of $M$ is said to be $\sigma$-dense in $M$ if $M / M^{\prime}$ is $\sigma$-torsion; it is said to be $\sigma$-closed in $M$ if $M / M^{\prime}$ is $\sigma$-torsionfree. The intersection of all $\sigma$-closed submodules of $M$ which contain $M^{\prime}$ is called the $\sigma$-closure of $M^{\prime}$ in $M$.

Received by the editors March 3, 1971.

AMS 1970 subject classifications. Primary 16A08; Secondary 16A50, 16A52.

Key words and phrases. Ring of left quotients, idempotent kernel functor, $\sigma$-torsionfree, $\sigma$-injective, $\sigma$-projective, $\sigma$-divisible.

(c) American Mathematical Society 1972 
A module ${ }_{R} N$ is called $\sigma$-injective if it has the following property: if ${ }_{R} M$ is any module and $M^{\prime}$ is any $\sigma$-dense submodule of $M$, then every $R$-homomorphism from $M^{\prime}$ to $N$ extends to a homomorphism from $M$ to $N$. For any module ${ }_{R} M$ the module of quotients $Q_{\sigma}(M)$ with respect to $\sigma$ is defined in the following way: let $Q_{\sigma}(M)$ be the $\sigma$-closure of $M / \sigma(M)$ in its injective envelope $E(M / \sigma(M))$. With this definition, $Q_{\sigma}(M)$ is $\sigma$-torsionfree and $\sigma$-injective, and $M / \sigma(M)$ is $\sigma$-dense in $Q_{\sigma}(M)$. In particular, $Q_{\sigma}(R)$ has a natural ring structure, and is called the ring of left quotients of $R$ with respect to $\sigma$. Every $\sigma$-torsionfree and $\sigma$-injective left $R$-module is a left module over $Q_{\sigma}(R)$, and moreover, the action of $Q_{\sigma}(R)$ on $M$ extends (uniquely) the action of $R$ on $M$.

If $R$ is commutative and $S$ is a multiplicatively closed set of elements of $R$, then the ring of quotients $R_{S}$ of $R$ with respect to $S$ has the property that $s \cdot R_{S}=R_{S}$ for all $s \in S$. Furthermore, any quotient of a direct sum of copies of $R_{S}$ shares this property of being " $S$-divisible". If $M$ is an $R$-module such that $s m \neq 0$ for all $s \in S$ and all $m \in M$, then $M$ is an $R_{S^{-}}$ module (extending the $R$-module structure) if and only if $M$ is " $S$ divisible". This motivates the following definition. (We use $M^{I}$ to denote the direct sum of modules indexed by the set $I$ and all isomorphic to ${ }_{R} M$.)

Definition 1. A module ${ }_{R} M$ is said to be $\sigma$-divisible if there exists an $R$-epimorphism $Q_{\sigma}(R)^{I} \rightarrow M \rightarrow 0$ for some index set $I$.

It is clear that ${ }_{R} M$ is $\sigma$-divisible if and only if for any $R$-homomorphism $0 \neq f: M \rightarrow N$ there exists a homomorphism $g: Q_{\sigma}(R) \rightarrow M$ such that $f g \neq 0$, so that $M$ is $\sigma$-divisible if and only if $Q_{\sigma}(R)$ generates $M$ in the categorical sense. It follows from this that the full subcategory of $\sigma$-divisible $R$ modules is cocomplete. (The reader is referred to [1] for this and further details.) We next give a definition which is shown by Proposition 3 to be equivalent to the corresponding definition in [2], but which is more convenient for our purposes.

Definition 2. A module ${ }_{R} P$ is $\sigma$-projective if for every diagram<smiles>[M][Y1](O)P</smiles>

with $M \rightarrow M^{\prime \prime}$ an epimorphism, $M$ and $M^{\prime \prime} \sigma$-torsionfree $R$-modules, and $M$ $\sigma$-injective, there is a homomorphism $P \rightarrow M$ making the diagram commutative.

Proposition 3. For a module ${ }_{R} P$ the following are equivalent:

(i) ${ }_{R} P$ is $\sigma$-projective.

(ii) For any epimorphism $M \rightarrow M^{\prime \prime} \rightarrow 0$ with $M$ and $M^{\prime \prime} \sigma$-torsionfree $R$ modules, and any homomorphism $P \rightarrow M^{\prime \prime}$, there is a $\sigma$-dense submodule $P^{\prime}$ 
of $P$ and a homomorphism $P^{\prime} \rightarrow M$ making the diagram

$$
\begin{aligned}
0 \rightarrow & P^{\prime} \rightarrow P \\
& \rightarrow \\
& \stackrel{\downarrow}{M} \rightarrow M^{\prime \prime} \rightarrow 0
\end{aligned}
$$

commutative.

Proof. (i) $\Rightarrow$ (ii) Given an epimorphism $M \rightarrow M^{\prime \prime} \rightarrow 0$ with $M$ and $M^{\prime \prime}$ $\sigma$-torsionfree, $Q_{\sigma}(M)$ and $Q_{\sigma}\left(M^{\prime \prime}\right)$ are extensions of $M$ and $M^{\prime \prime}$ respectively, and $M \rightarrow M^{\prime \prime}$ extends to $Q_{\sigma}(M) \rightarrow Q_{\sigma}\left(M^{\prime \prime}\right)$. If $N$ is the image of this homomorphism, this gives the diagram

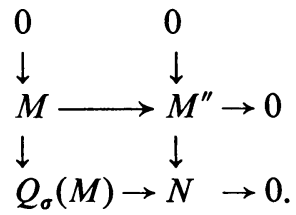

If $P \rightarrow M^{\prime \prime}$ is any homomorphism, then if ${ }_{R} P$ is $\sigma$-projective, $P \rightarrow M^{\prime \prime} \rightarrow N$ lifts to $f: P \rightarrow Q_{\sigma}(M)$ because $Q_{\sigma}(M)$ is $\sigma$-injective and both $Q_{\sigma}(M)$ and $N$ are $\sigma$-torsionfree. Let $P^{\prime}=f^{-1}(M)$. Since $M$ is $\sigma$-dense in $Q_{\sigma}(M)$, it follows that $P^{\prime}$ is $\sigma$-dense in $P$, and thus $f$ restricted to $P^{\prime}$ gives the required homomorphism.

(ii) $\Rightarrow$ (i) If $P$ satisfies condition (ii) and the diagram

$$
\begin{gathered}
P \\
\stackrel{\pi}{\longrightarrow} M^{\prime \prime} \longrightarrow 0
\end{gathered}
$$

is given with $M \sigma$-injective and $M$ and $M^{\prime \prime}$ both $\sigma$-torsionfree, then there exists a $\sigma$-dense submodule $P^{\prime}$ of $P$ and a homomorphism $g^{\prime}: P^{\prime} \rightarrow M$ such that $g^{\prime}=f i$, where $i: P^{\prime} \rightarrow P$ is the inclusion. Since $M$ is $\sigma$-injective and $P^{\prime}$ is $\sigma$-dense in $P, g^{\prime}$ extends to $g: P \rightarrow M$, with $g^{\prime}=g i$. But then $f i=\pi g i$, and it follows from the fact that $P^{\prime}$ is $\sigma$-dense in $P$ and $M^{\prime \prime}$ is $\sigma$-torsionfree that $f=\pi g$.

The next theorem is our main result. It gives a condition which characterizes $\sigma$-torsionfree, $\sigma$-divisible $R$-modules and which shows that for $\sigma$ torsionfree modules the notion of $\sigma$-divisible is a generalization of that of $\sigma$-injective. Furthermore, it shows that among $\sigma$-torsionfree $R$-modules the $\sigma$-divisible modules are precisely those whose $R$-module structure can be extended to a $Q_{\sigma}(R)$-module structure.

THEOREM 4. Let ${ }_{R} M$ be $\sigma$-torsionfree. Then the following conditions are equivalent:

(i) $M$ is $\sigma$-divisible.

(ii) If $N^{\prime}$ is a finitely generated, $\sigma$-projective and $\sigma$-dense submodule of a 
module ${ }_{R} N$, then every $R$-homomorphism from $N^{\prime}$ to $M$ extends to a homomorphism from $N$ to $M$.

(iii) The $R$-module structure of $M$ can be extended uniquely to give $M$ the structure of a left $Q_{\sigma}(R)$-module.

Proof. (i) $\Rightarrow$ (ii) Let $N^{\prime}$ be a finitely generated, $\sigma$-projective and $\sigma$ dense submodule of a module ${ }_{R} N$ and $f: N^{\prime} \rightarrow M$ be an $R$-homomorphism. If $M$ is $\sigma$-divisible, then there exists an $R$-epimorphism $\pi: Q_{\sigma}(R)^{I} \rightarrow M$ for some index set $I$. Since $N^{\prime}$ is finitely generated, so is $f\left(N^{\prime}\right)$, and thus $f\left(N^{\prime}\right)$ must be contained in the image of a finite number of summands of $Q_{\sigma}(R)^{I}$. Letting $M^{\prime}$ be this image of a direct sum of finitely many copies of $Q_{\sigma}(R)$, we have the following diagram:

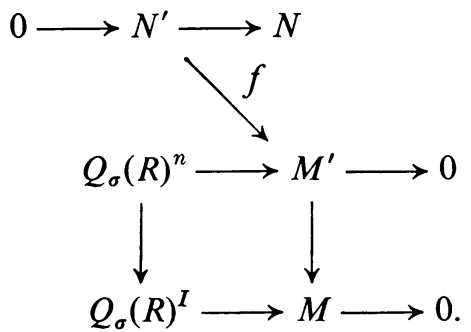

But now $Q_{\sigma}(R)^{n}$ is $\sigma$-injective since it is a direct sum of finitely many $\sigma$ injective modules, and in addition, both $Q_{\sigma}(R)^{n}$ and $M^{\prime}$ are $\sigma$-torsionfree. It then follows from the assumption that $N^{\prime}$ is $\sigma$-projective that $f$ can be lifted to a homomorphism $f^{\prime}: N \rightarrow Q_{\sigma}(R)^{n}$. Because $N^{\prime}$ is $\sigma$-dense in $N$ and $Q_{\sigma}(R)^{n}$ is $\sigma$-injective, $f^{\prime}$ can be extended to $g: N \rightarrow Q_{\sigma}(R)^{n} \rightarrow Q_{\sigma}(R)^{I}$, and $\pi g$ gives the required extension of $f$ to $N$.

(ii) $\Rightarrow$ (iii) We first observe that if ${ }_{R} P$ is a projective $R$-module, then $P / \sigma(P)$ is $\sigma$-projective, since any $R$-homomorphism from $P$ into a $\sigma$ torsionfree module factors through $P / \sigma(P)$. In particular, $R / \sigma(R)$ is $\sigma$ projective. Since $M$ is $\sigma$-torsionfree, for each $m \in M$ the homomorphism from $R$ to $M$ defined by $r \mapsto r m$ factors through $R / \sigma(R)$, and then if $M$ satisfies condition (ii) this extends to $Q_{\sigma}(R)$, since $R / \sigma(R)$ is finitely generated, $\sigma$-projective, and $\sigma$-dense in $Q_{\sigma}(R)$. The extension is unique since $M$ is $\sigma$-torsionfree. That this is the required $Q_{\sigma}(R)$-module structure on $M$ can be easily verified.

(iii) $\Rightarrow$ (i) If $M$ is a $Q_{\sigma}(R)$-module, then it is a quotient of a free $Q_{\sigma}(R)$ module. Viewing $M$ as an $R$-module under the induced $R$-module structure, this shows that $M$ is $\sigma$-divisible. It follows that if ${ }_{R} M$ has a $Q_{\sigma}(R)$-module structure which extends the $R$-module structure, then $M$ is $\sigma$-divisible.

We recall that Goldman calls $\sigma$ Noetherian if the following property is satisfied: if $A_{1} \subseteq A_{2} \subseteq \cdots$ is an ascending chain of left ideals of $R$ whose union is $\sigma$-dense in $R$, then for some $n, A_{n}$ is $\sigma$-dense in $R$. Any direct sum 
of $\sigma$-torsionfree, $\sigma$-injective $R$-modules is $\sigma$-injective if and only if $\sigma$ is Noetherian. If we assume that $\sigma$ is Noetherian, then condition (ii) of Theorem 4 can be simplified somewhat.

Proposition 5. Let ${ }_{R} M$ be $\sigma$-torsionfree, and assume that $\sigma$ is Noetherian. Then the following are equivalent:

(i) $M$ is $\sigma$-divisible.

(ii) If $N^{\prime}$ is a $\sigma$-projective and $\sigma$-dense submodule of ${ }_{R} N$, then every $R$ homomorphism from $N^{\prime}$ to $M$ extends to a homomorphism from $N$ to $M$.

Proof. It is immediate from the previous theorem that (ii) $\Rightarrow$ (i). Conversely, assume that $\sigma$ is Noetherian and $M$ is $\sigma$-torsionfree and $\sigma$ divisible. Since $\sigma$ is Noetherian, any direct sum of copies of $Q_{\sigma}(R)$ is $\sigma$ injective, so any $R$-homomorphism from a $\sigma$-projective module $N^{\prime}$ into $M$ can be lifted to the module $Q_{\sigma}(R)^{I}$ lying above $M$. The proof then follows as before.

The idempotent kernel functor $\sigma$ is said by Goldman to have property (T) if $Q_{\sigma}(M)$ is isomorphic to $Q_{\sigma}(R) \otimes_{R} M$ for all modules ${ }_{R} M$. Such kernel functors can be characterized using the notion of $\sigma$-divisibility.

THEOREM 6. The following conditions are equivalent:

(i) $\sigma$ has property $(\mathrm{T})$.

(ii) Every $\sigma$-torsionfree, $\sigma$-divisible left $R$-module is $\sigma$-injective.

Proof. Theorems 4.4 and 4.5 of [2] show that $\sigma$ has property (T) if and only if (a) every direct sum of $\sigma$-torsionfree, $\sigma$-injective modules is $\sigma$-injective and (b) every $\sigma$-torsionfree quotient of a $\sigma$-torsionfree, $\sigma$ injective module is $\sigma$-injective. The proof then follows directly from the definition of $\sigma$-divisible and the fact that all direct sums and quotients of $\sigma$-divisible modules are again $\sigma$-divisible.

\section{REFERENCES}

1. J. A. Beachy, Generating and cogenerating structures, Trans. Amer. Math. Soc. 158 (1971), 75-92.

2. O. Goldman, Rings and Modules of quotients, J. Algebra 13 (1969), 10-47. MR 39 \#6914.

3. J. Lambek, Torsion theories, additive semantics, and rings of quotients, Lecture Notes in Math., no. 177, Springer-Verlag, Berlin and New York, 1971.

Department of Mathematics, Northern Illinois University, DeKalb, Illinois 60115 